\title{
Impact du développement des palmeraies familiales sur la déforestation et dans les ménages au Cameroun
}

\author{
Assoumou M. R. MEZUI ${ }^{1 *}$, Mate J. P. MWERU ${ }^{2}$ et I. R. TCHOUAMO ${ }^{3}$ \\ ${ }^{I}$ IRAD, B.P. 2067, Yaoundé, Cameroun. \\ ${ }^{2}$ ERAIFT/UNESCO. \\ ${ }^{3}$ Université de Dschang, Faculté d'Agronomie et des Sciences Agricoles, B.P. 245, Dschang, Cameroun. \\ *Auteur correspondant, E-mail: remy_assoumou@yahoo.com; assoumou.mry3@gmail.com
}

\section{RESUME}

Une étude a été conduite au sein de 208 exploitants familiaux dans le but d'estimer l'impact de l'expansion de l'élaéiculture sur la déforestation et les revenus des ménages au Cameroun. La méthode de l'Effet Moyen du Traitement a été utilisée dans une modélisation Probit. Les résultats montrent que le taux de déforestation causé par les producteurs ayant obtenu le matériel végétal sélectionné (Tenera) d'une source formelle, est significativement supérieur à celui des producteurs qui se sont approvisionnés dans des sources informelles. Les facteurs qui déterminent cette déforestation sont l'âge et la politique agricole. L'âge est en relation étroite avec l'aptitude à posséder les facteurs de production (terre, capital et travail) qui deviennent plus rares lorsque le producteur est jeune, c'est-à-dire moins de 30 ans. Dans les ménages des exploitants familiaux, l'élaéiculture contribue à plus de 50\% au revenu agricole annuel. Cette activité donne également de l'huile de palme nécessaire pour la consommation familiale. Ces résultats ont déclenché une réflexion sur l'opportunité d'élaborer un outil d'aide aux politiques agricoles prenant en compte les objectifs d'accroître la production nationale d'huile de palme et celui de gérer durablement des ressources forestières.

(C) 2012 International Formulae Group. All rights reserved.

Mots clés: Déforestation, exploitants familiaux, impact, palmier à huile.

\section{INTRODUCTION}

La déforestation a souvent été attribuée à une gamme de causes (Estrada Porrúra et al., 2007). Ces causes s'étendent du manque de récompense de la part du marché du carbone pour avoir conservé les forêts, à l'attribution inadéquate des droits de propriété, à l'échec des politiques et aux mauvaises pratiques de gestion (Otsuka and Place, 2001). Toutes ces causes ont chacune une part de vérité dans la mesure où la déforestation est liée aux activités humaines.
La dégradation est souvent définie comme une perte de la biomasse (baisse du stock de carbone) ne conduisant pas à la disparition totale du couvert forestier. Alors que la déforestation est la disparition du couvert forestier conséquente à un changement d'usage des terres sous couvert forestier (Doumenge, 1998).

$\mathrm{Au}$ Cameroun, les causes de la déforestation et de la dégradation des forêts sont aussi multiples et des controverses apparaissent souvent lorsqu'il faut les classer par ordre d'importance. Les causes 
fréquemment citées sont: la croissance démographique en zone urbaine, les pratiques agricoles (cultures vivrières sur brûlis, cultures de rente), l'exploitation illégale du bois d'œuvre, l'exploitation forestière dans les Unités Forestières d'Aménagement (UFA), les projets de développement du secteur minier, la construction des infrastructures routières dans le cadre du désenclavement du pays et les feux de brousse utilisés par les chasseurs et les éleveurs de gros bétail (Amougou, 2010 ; Delacote, 2008 ; World Rainforest Movment, 2007 ; FAO, 2007 ; Rossi et André, 2006 ).

En ce qui concerne les pratiques agricoles, il est rapporté qu'en Amérique latine et en Asie, la déforestation est causée de manière croissante par la conversion aux plantations industrielles de soja, canne à sucre et palmier à huile (Kanninen et al., 2007). En effet, s'il est vrai que l'huile de palme présente des avantages économiques indéniables comme de hauts rendements en huile végétale, un faible coût de revient et la création d'emplois, il n'en demeure pas moins vrai que les palmeraies sont considérées comme les principales causes de la déforestation en Malaisie et en Indonésie ou environ 6 millions d'hectares sont consacrés à l'élaéiculture (Fitzherbert et al., 2008). En plus, l'exploitation des palmeraies contribue à la pollution de l'environnement (sols, eaux et air).

L'hypothèse générale qui sous-tend cette étude est que l'expansion de l'élaéiculture suite à la grande diffusion et l'adoption de la semence sélectionnée de palmier à huile au sein des producteurs familiaux camerounais, conduit à l'augmentation des superficies des palmeraies et par ricochet à la déforestation. Plus spécifiquement, l'impact causé par un producteur choisi au hasard dans toute la population des élaéiculteurs, est différent en fonction du type de source d'approvisionnement en matériel végétal sélectionné. Les sources formelles sont généralement le Programme de Développement des Palmeraies Villageoise
(PDPV) ou les centres de recherche comme Pamol Plantation Limited et l'Institut de Recherche Agricole pour le Développement (IRAD), Centre de Recherche Spécialisé sur le Palmier à huile (CEREPAH) de la Dibamba. Les sources informelles sont les pépiniéristes, d'autres producteurs et le ramassage sous les palmeraies industrielles. Une autre hypothèse est que la déforestation (ou l'augmentation des superficies des palmeraies), est déterminée par certains facteurs sociaux, économiques et environnementaux.

Les objectifs de cette étude sont d'estimer les paramètres et identifier les déterminants d'impact de l'expansion de l'élaéiculture camerounaise sur la déforestation d'une part et les revenus des ménages d'autre part. La méthode ayant permis d'atteindre ces objectifs est présentée dans le paragraphe suivant.

\section{MATERIEL ET METHODES}

La méthode d'échantillonnage est stratifiée au niveau régional et raisonné. L'échantillon des producteurs de palmier à huile est stratifié au niveau des trois régions du Cameroun comprises entre les points de coordonnées géographiques ( $4^{\circ} 31^{\prime} 23,4^{\prime}$ ' $\mathrm{N}$ $\left.9^{\circ} 21^{\prime} 21,5^{\prime}, \mathrm{E}\right)$ et $\left(4^{\circ} 38^{\prime} 50,0^{\prime}, \mathrm{N}-12^{\circ} 22^{\prime}\right.$ $53,7^{\prime}$ 'E), et les points de coordonnées $\left(2^{\circ} 16^{\prime}\right.$ 16,4 '’ $\left.\mathrm{N}-12^{\circ} 19^{\prime} 31,1^{\prime \prime} \mathrm{E}\right)$ et $\left(2^{\circ} 14^{\prime} 18,1^{\prime \prime} \mathrm{N}\right.$ - $9^{\circ} 51^{\prime} 27,3$ ' $\left.\mathrm{E}\right)$. Toute cette zone est très favorable à la culture du palmier à huile.

Un choix raisonné de 208 enquêtés a été effectué parmi les producteurs de palmier à huile des trois régions dont 46 dans la région du Littoral, 42 dans la région du Centre et 120 dans la région du Sud. Le seul critère de sélection d'un enquêté était la possession d'une palmeraie. Les déterminants d'impact sur la déforestation ont été évalués au sein de toute cette population dans un modèle Probit. Cependant l'impact de l'expansion de l'élaéiculture dans les ménages des exploitants familiaux a été évalué au sein de la sous population de 180 producteurs seulement à cause de certaines données d'enquête manquantes. 
La source d'approvisionnement en matériel végétal est considérée comme la variable instrumentale (Z). En effet, les producteurs peuvent s'en procurer auprès des différentes sources formelles (dans ce cas $\mathrm{Z}=$ $1)$; ou informelles $(Z=0)$ qui sont à leur portée.

Comme les producteurs de palmier à huile n'ont pas de contrôle sur le type de source d'approvisionnement en matériel végétal sélectionné Tenera, l'intervention est de source extérieure. En plus, l'innovation Tenera n'a pas fait l'objet de diffusion dans à travers des essais en milieu paysan en vue susciter son adoption par des participants. Par conséquent, l'intervention est de type exogène.

La population des producteurs de palmier à huile est $(\mathrm{D}=208)$. Elle se subdivise en deux sous-population, c'est-à-dire ceux qui se sont approvisionnés dans des sources formelles $\left(D_{1}=131\right)$ et ceux qui se sont approvisionnés dans des sources informelles $\left(\mathrm{D}_{0}=77\right)$.

A cause de la diversité des conditions sociales, économiques et environnementales dans les trois régions d'étude, tous les producteurs n'ont pas la même chance de s'approvisionner dans un même type de source en matériel végétal sélectionné du palmier à huile. Par conséquent l'instrument (Z) est distribué de manière non-aléatoire au sein de toute la population. Nous dirons en substance que l'intervention est de « Type II » (Diagne and Demont, 2007).

La méthode d'estimation des paramètres d'impact sera donc celle de l'Effet Moyen de Traitement (Average Treatment Effect 'ATE'). ATE est une approche naïve qui permet d'estimer l'impact d'une intervention causé par une personne sélectionnée au hasard au sein la population (Caliendo and Hujer, 2005).

L'état de traitement (ou intervention) est une fonction de l'instrument $\mathrm{Z} \in\{0,1\}$. Ainsi, deux résultats potentiels peuvent être observés sous état de traitement : D'abord D = $\mathrm{D}_{1}$ si $\mathrm{Z}=1$, ou $\mathrm{D}=\mathrm{D}_{0}$ si $\mathrm{Z}=0$.
Le résultat observé sous état de traitement est : $\mathrm{D}=\mathrm{Z} * \mathrm{D}_{1}+(1-\mathrm{Z}) * \mathrm{D}_{0}$

L'effet moyen du traitement se calcule alors selon l'équation suivante :

$\mathrm{ATE}=\mathrm{E}\left(\mathrm{Y}_{1}-\mathrm{Y}_{0}\right)=\mathrm{E}\left(\mathrm{Y}_{1}-\mathrm{Y}_{0} \mid \mathrm{D}=1\right)^{*}$

$\mathrm{P}(\mathrm{D}=1)+\mathrm{E}\left(\mathrm{Y}_{1}-\mathrm{Y}_{0} \mid \mathrm{D}=0\right) * \mathrm{P}(\mathrm{D}=1)$

Avec :

D: variable $\mathrm{du}$ statut $\mathrm{du}$ traitement/intervention observé $\varepsilon\{0,1\}$

$\mathrm{P}$ : Probabilité de sélection d'un individu au sein de la population

$(D=1)$ : État sous intervention/traitement

$(\mathrm{D}=0)$ : État sous non intervention/traitement

$\mathrm{Y}_{1}$ : Résultat potentiel sous état d'intervention / traitement

$\mathrm{Y}_{0}$ : Résultat potentiel sous état de non intervention / traitement

$\mathrm{Y}$ : Variable de résultat observée $=\mathrm{D}^{*} \mathrm{Y} 1+$ $(1-\mathrm{D}) * \mathrm{Y} 0$

$\mathrm{ATE}_{1}$ : c'est l'Effet Moyen du Traitement sur les traités, c'est-à-dire le taux moyen d'impact causé par un individu sélectionné au hasard dans la sous population de ceux qui ont subit l'intervention uniquement ou bien ceux qui se sont approvisionnés dans une source formelle $\left(D_{1}\right): \mathrm{ATE}_{1}=\mathrm{E}\left(\mathrm{Y}_{1}-\mathrm{Y}_{0} \mid \mathrm{D}=1\right)$

$\mathrm{ATE}_{0}$ : c'est l'Effet Moyen du Traitement sur les non-traités, c'est-à-dire le taux moyen d'impact causé par un individu sélectionné au hasard dans la sous population de ceux qui n'ont pas subit l'intervention; ou encore ceux qui se sont approvisionnés dans une source informelle $\left(\mathrm{D}_{0}\right)$ :

$\mathrm{ATE}_{0}=\mathrm{E}\left(\mathrm{Y}_{1}-\mathrm{Y}_{0} \mid \mathrm{D}=0\right)$

MD : (Mean Difference) : C'est la différence de moyennes des résultats observés des traités et des non-traités :

$\mathrm{MD}=\mathrm{E}(\mathrm{Y} \mid \mathrm{D}=1)-\mathrm{E}(\mathrm{Y} \mid \mathrm{D}=0)$

PSB (Population Selection Bias) est le biais de sélection de la population, encore appelé Score de propension:

$\mathrm{PSB}=\mathrm{ATE}_{1}-\mathrm{ATE}$

Dans le modèle, la variable dépendante (DEFOREST) mesure la superficie en 
déforestation. Ce facteur impacté peut être fonction : i) des caractéristiques personnelles du producteur (âge, sexe, statut matrimonial, disponibilité de la main d'œuvre familiale, objectif de production, disponibilité des terres, niveau de revenu); ii) des conditions environnementales (zone agro écologique) (REGION); iii) de la politique agricole en faveur de la diffusion et de l'adoption de l'innovation (TENERA).

\section{RESULTATS ET DISCUSSION}

Trois catégories de résultats ont été produites dans cette étude. Les premiers résultats (Tableau 1), sont relatifs à l'estimation des paramètres d'impact de l'expansion de l'élaéiculture sur la déforestation selon les conditions d'expositions des producteurs familiaux aux deux types de sources d'approvisionnement en matériel végétal sélectionné du palmier à huile en l'occurrence les sources formelles (IRAD et Pamol) et les sources informelles (pépiniéristes et autres). La deuxième catégorie de résultats (Tableau 2), concerne les facteurs qui déterminent cet impact environnemental. Le troisième résultat estime l'impact socio-économique du développement de l'élaéiculture dans les ménages des exploitants familiaux.

\section{Estimation des paramètres d'impact de l'expansion de l'élaéicutlture sur la déforestation}

Les paramètres d'impact de l'expansion de l'élaéiculture sur la déforestation ont été estimés par la méthode d'Effet Moyen du Traitement (ATE) paramétrique des moindres carrés ordinaires (OLS) et les résultats sont présentés dans le Tableau 1 suivant.

Selon le Tableau 1, le taux de déforestation ou bien la proportion réelle d'augmentation des superficies des palmeraies d'un individu choisi au hasard dans la souspopulation de ceux qui se sont approvisionnés dans une source formelle de matériel végétal sélection (appelés «traités »), est hautement significative $\left(\mathrm{ATE}_{1}=19,42 \% ; \mathrm{p}=0,001\right)$. De même, le taux potentiel de déforestation causé par un individu sélectionné au hasard au sein de toute la population des producteurs de palmier à huile est aussi significatif ATE = $12,26 \% ; \mathrm{p}=0,000)$. Ainsi, la propension à la déforestation est hautement significative (PSB $=7,15 \% ; \mathrm{p}=0,000$ ).

Ce résultat signifie que, l'opportunité que certains producteurs ont pour s'approvisionner en intrants agricoles (matériel végétal sélectionné et engrais) dans une source formelle, augmente significativement les surfaces des palmeraies familiales. Autrement dit, une politique agricole qui subventionne l'approvisionnement des producteurs familiaux en intrants agricoles (semences améliorées et engrais) augmentera significativement le taux de déforestation. Ce résultat correspond bien aux observations faites par (Besley and Case, 1993; Sascha and Andres, 2002; Dimara, and Skuras, 2003) sur l'adoption des innovations et la réduction des émissions de gaz à effet de serre en zones forestières. Ce résultat est confirmé quand nous observons que le taux moyen de déforestation causée par tous les traités est supérieur à celui causé par les non-traités ; car la différence des moyenne est significativement positive avec (MD = $22,94 \% ; \mathrm{p}=0,001)$.

\section{Déterminants d'impact sur la déforestation \\ Dans le Tableau 2, l'analyse de} variance par la méthode des moindres carrés ordinaires (OLS) est significative $(\mathrm{F}(19,188)$ $=30,24$ et $\mathrm{p}=0,000)$. Ce qui signifie que le modèle de régression Probit présenté dans le Tableau 3, est bon pour identifier les déterminants d'impact de la diffusion et adoption du matériel végétal sélectionné Tenera sur la déforestation (ou augmentation des superficies des palmeraies familiales) dans l'échantillon d'étude.

Selon le Tableau 2, la diffusion et l'adoption de l'hybride Tenera de palmier à huile (TENERA) à elle seule, n'a pas un impact significatif sur la déforestation $(\mathrm{C}=$ 
0,071; $\mathrm{p}=0,815)$. Cependant, cette diffusion et adoption a un impact significatif lors qu'elle est en combinaison avec d'autres facteurs comme l'âge de l'exploitant (AGE) et la politique agricole en vigueur (ENCAD).

En effet, seules les deux variables, âge $\mathrm{du}$ producteur (AGE) et la politique agricole en vigueur (ENCAD) prises individuellement, influencent positivement ou négativement la déforestation (DEFOREST) de manière significative. Leurs coefficients sont respectivement $(\mathrm{C}=0,0107 ; \mathrm{p}=0,000)$ et $(\mathrm{C}=-0,6578 ; \mathrm{p}=0,000)$.

Par ailleurs, l'interaction entre l'adoption de l'hybride Tenera (TENERA) et les variables explicatives du modèle telles que (AGE, ENCAD et DEFOREST), influence significativement l'augmentation des superficies des palmeraies familiales. Leurs coefficients sont respectivement de $(\mathrm{C}=$ $0,0106 ; \mathrm{p}=0,002) ; \quad(\mathrm{C}=0,6477 ; \mathrm{p}=$ $0,000)$ et $(C=0,9982 ; p=0,000)$.

$\mathrm{Si}$ le facteur (AGE) pris individuellement, est déterminant pour l'augmentation des superficies des palmeraies familiales, c'est parce que l'élaéiculture exige non seulement une main d'œuvre non négligeable, mais aussi un investissement considérable de l'ordre de 578000 FCFA (1,084.91 USD) par hectare et pour les trois premières années de plantation dans les systèmes de production des petits producteurs utilisant une main d'œuvre familiale et peu ou pas d'engrais (Lebailly et Tenchou, 2009). En plus, lors d'un tel investissement, $65 \%$ des coûts en première année correspondent à l'acquisition du matériel végétal uniquement. Par contre, la période de récupération de l'investissement est de huit ans ; parce qu'une palmeraie conduite avec un matériel végétal sélectionné n'entre en production qu'à partir de la troisième année.

De nos jours, la population camerounaise est composée de $72 \%$ de jeunes dont l'âge est inférieur à 30 ans. Plus de la moitié de ces jeunes (53\%) vivent dans des centres urbains (BUCREP, 2010). Ces jeunes vivent généralement en dessous du seuil de pauvreté (moins d'un dollar US par jour) et se trouvent incapables de créer des palmeraies en milieu rural, parce que les adultes et fonctionnaires qui sont plus nantis, accentuent leur course effrénée à l'occupation des terres, parfois pour un objectif de bornage. En substance, ce sont les facteurs de production (terre, capital et travail) qui déterminent l'augmentation des superficies des palmeraies familiales au Cameroun et ce sont surtout les jeunes qui n'en disposent pas.

Le second facteur qui détermine individuellement l'augmentation des superficies des palmeraies familiales c'est la politique agricole. Notons que la politique agricole peut être favorable ou non au développement de l'élaéiculture au Cameroun. Le signe négatif porté par ce facteur dans le Tableau 2, signifie que la politique agricole actuelle n'a pas un impact positif sur l'augmentation des superficies des palmeraies familiales. Cette politique a été ralentie dans la quasi-totalité des filières agricoles depuis le début de la crise économique survenue au Cameroun au cours des années 1987. Cet encadrement agricole subventionnait la fourniture des intrants agricoles (semences, engrais, pesticides), l'octroi des crédits à travers les institutions comme le Fonds National de Développement Rural (FONADER) et le suivi des activités de production des agriculteurs surtout dans les filières des cultures d'exportation à l'instar de la filière Cacao-café.

Ce n'est que depuis l'an 2003, que le Programme de Développement des Palmeraies Villageoises (PDPV) a été lancé pour appuyer l'approvisionnement des producteurs en intrants agricoles (semences sélectionnées et engrais). Ce programme évolue timidement. Pour ce faire, entre 2003 et 2010, le PDPV n'a favorisé l'accroissement des superficies des palmeraies familiales que d'environ 6500 hectares en encadrant près de 5000 agriculteurs qui ont reçu gratuitement de jeunes plants de palmier sélectionné (hybride 
Tenera), y compris de l'engrais au démarrage de la plantation (UNEXPALM, 2010). Cette politique mérite d'être améliorée pour une augmentation effective des superficies des palmeraies familiales et par extension, l'augmentation de la production d'huile de palme au Cameroun.

Les facteurs âge et politique agricole peuvent donc être considérés comme les grands leviers pour le développement efficace et durable de l'élaéiculture au Cameroun. Un programme de développement de l'élaéiculture sera plus efficace si ses objectifs de subventionner la création des palmeraies est surtout orientée vers les jeunes qui sont plus les plus démunis mais aussi plus nombreux. Un tel programme favoriserait un accroissement substantiel de la production d'huile de palme dans un moyen terme. Cependant, le développement de l'élaéiculture nécessite la destruction d'importantes ressources forestières et participe à la pollution de l'environnement. Pour ce faire, une politique axée sur la relance de la compétitivité de la filière palmier à huile sera durable si la poursuite de ses objectifs prend en compte la préservation des ressources naturelles et la protection de l'environnement. Avant de proposer des solutions concrètes sur cette situation contrastée, trouvons dans le paragraphe suivant l'impact socioéconomique de la diffusion et l'adoption de la variété sélectionnée de palmier à huile dans les ménages des producteurs ?

Impact socio économique de l'adoption de Tenera

L'adoption du palmier à huile sélectionné (Tenera) a eu un impact économique dans les ménages des producteurs puisque $52 \%$ de répondants affirment que l'élaéiculture contribue à plus de 50\% dans leurs revenus annuels. En plus, les trois principales motivations à la production du palmier à huile par les exploitants familiaux sont: la satisfaction des besoins de consommation et de commercialisation
(21,7\%), l'obtention d'une meilleure production d'huile végétale $(8,9 \%)$ et la proximité des huileries dans certains villages $(6,7 \%)$.

Les trois principales contraintes signalées par les répondants sont: la rareté des magasins de vente des intrants agricoles $(21,1 \%)$, le manque de structures de financement agricole $(13,3 \%)$ et le manque des services d'encadrement technique en agriculture $(11,7 \%)$.

Comme l'élaéiculture contribue à plus de $50 \%$ dans les revenus annuels des ménages des exploitants familiaux et les principales motivations à cette activité agricole sont la consommation et la commercialisation, nous pouvons confirmer que c'est une activité rémunératrice qui nourri bien l'homme. Cette activité agricole peut donc améliorer la suffisance alimentaire et lutter contre la pauvreté dans les pays en développement comme le Cameroun.

En plus, si les deux principales contraintes d'approvisionnement en intrants agricoles et de financement des activités de production peuvent être maîtrisées dans le cadre d'un programme de développement durable de l'élaéiculture au Cameroun, beaucoup de jeunes producteurs peuvent entrer dans la filière. On assistera inéluctablement à une augmentation des superficies des palmeraies familiales, à l'amélioration des revenus des ménages mais aussi à l'augmentation du taux potentiel de déforestation.

Les contraintes soulevées par les producteurs enquêtés (la rareté des magasins de vente des intrants agricoles, le manque des structures de financement agricole et le manque des services d'encadrement technique en agriculture), confirment la proposition sur la mise œuvre d'une politique agricole adéquate au développement efficace et durable de l'élaéiculture au Cameroun. 


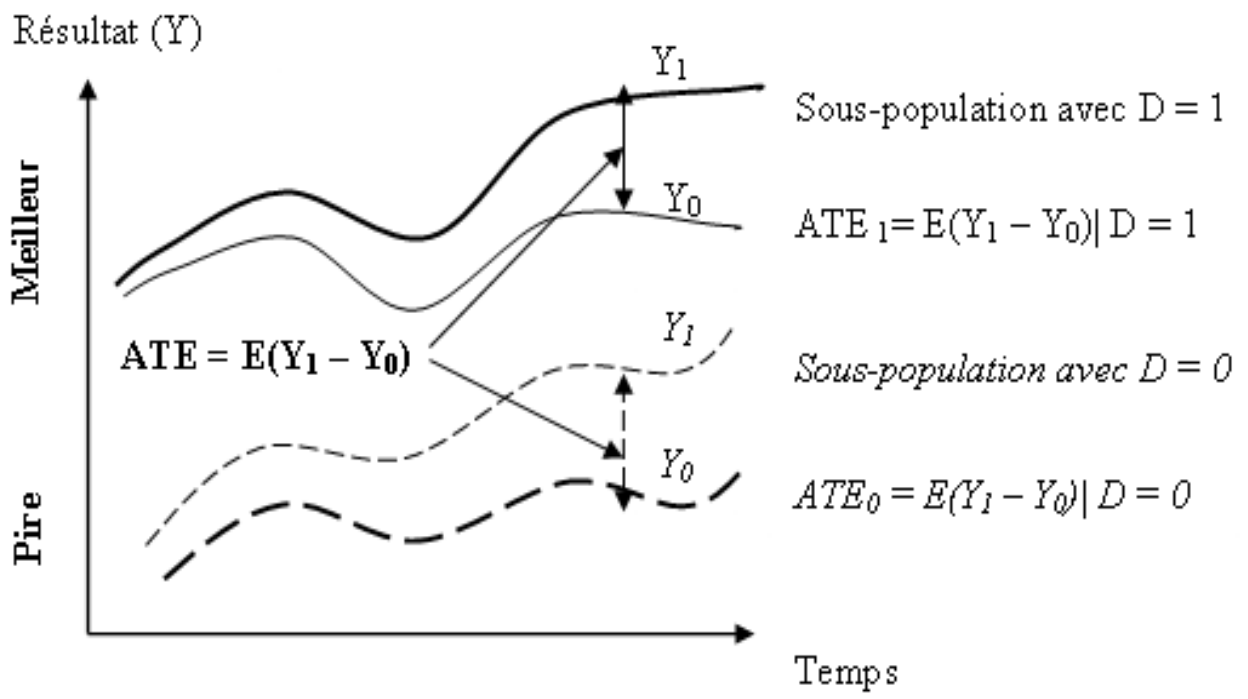

Figure 1: Paramètres d'impact au sein de la population.

Tableau 1: Paramètres d'impact par la méthode ATE paramétrique : OLS.

\begin{tabular}{|c|c|c|c|c|}
\hline Paramètre & Estimation & Erreur Std. Robuste & $\mathbf{Z}$ & $\mathbf{P}>|\mathbf{z}|$ \\
\hline ATE & $0,1226508^{* *}$ & 0,0377781 & 3,25 & 0,001 \\
\hline $\mathrm{ATE}_{1}$ & $0,1941947 * * *$ & 0,0386019 & 5,03 & 0,000 \\
\hline $\mathrm{ATE}_{0}$ & 0,0009334 & 0,0401974 & 0,02 & 0,981 \\
\hline PSB & $0,0715438 * * *$ & 0,0080213 & 8,92 & 0,000 \\
\hline \multicolumn{5}{|l|}{ Observés } \\
\hline MD & $0,2294042 * *$ & 0,0697177 & 3,29 & 0,001 \\
\hline mo_N $N_{1}$ & $0,7099237 * * *$ & 0,0398405 & 17,82 & 0,000 \\
\hline mo_ $\mathrm{N}_{0}$ & $0,4805195^{* * *}$ & 0,0572128 & 8,40 & 0,000 \\
\hline
\end{tabular}

Tableau 2: Déterminants d'impact sur la déforestation.

\begin{tabular}{|c|c|c|c|}
\hline Source & Somme des Carrés & dl & Carrés Moyens \\
\hline Modèle & 36,7310823 & 19 & 1,93321486 \\
\hline Erreur & 12,0189177 & 188 & 0,063930413 \\
\hline Total & 48,75 & 207 & 0,235507246 \\
\hline \multicolumn{4}{|c|}{$\begin{array}{ll}\text { Nombre d'observations } & =208 \\
\mathrm{~F}(19,188) & =30,24 \\
\text { Prob }>\mathrm{F} & =0,0000 \\
\mathrm{R}^{2} & =0,7535 \\
\mathrm{R}^{2} \text { Ajusté } & =0,7285 \\
\sqrt{ } \mathrm{CME} & =0,2528\end{array}$} \\
\hline
\end{tabular}




\begin{tabular}{|c|c|c|}
\hline Facteur & Coefficient & $\mathbf{P}>|\mathbf{t}|$ \\
\hline \multicolumn{3}{|l|}{ DEFOREST } \\
\hline TENERA & $0,0709651^{\mathrm{ns}}$ & 0,815 \\
\hline DISPOTER & 0,0184846 & 0,769 \\
\hline REGION & 0,0168375 & 0,798 \\
\hline SEXE & 0,0664058 & 0,431 \\
\hline AGE & $0,0106735^{* *}$ & 0,000 \\
\hline STATMAT & $-0,0752065$ & 0,403 \\
\hline EDUC & 0,0511757 & 0,118 \\
\hline ACTIVITE & 0,0281939 & 0,505 \\
\hline TAILLEMEN & 0,0024458 & 0,731 \\
\hline ENCAD & $-0,6577915^{* *}$ & 0,000 \\
\hline REGION x TENERA & $-0,0190812$ & 0,815 \\
\hline SEXE x TENERA & $-0,0657739$ & 0,569 \\
\hline AGE $x$ TENERA & $-0,0105849 * *$ & 0,002 \\
\hline STATMAT x TENERA & 0,0699823 & 0,521 \\
\hline EDUC $x$ TENERA & $-0,0549253$ & 0,159 \\
\hline DISPOTER x TENERA & $-0,0187875$ & 0,810 \\
\hline TAILLEMEN x TENERA & $-0,0026213$ & 0,753 \\
\hline ENCAD x TENERA & $0,6476969 * * *$ & 0,000 \\
\hline DEFOREST $\mathrm{x}$ TENERA & $0,998164 * * *$ & 0,000 \\
\hline CONST & $-0,061355$ & 0,792 \\
\hline \multicolumn{3}{|c|}{${ }^{\text {ns }}:$ non significatif $; * *$ : significatif ; $* * *$ : hautement significatif au seuil $\alpha=5 \%$} \\
\hline Test de Wald sans interaction $\mathrm{F}$ & 12, $52 \quad$ Prob $>F=$ & \\
\hline
\end{tabular}

Tableau 3: Impact de l'adoption de Tenera dans les ménages des producteurs de palmier à huile.

\begin{tabular}{llcc}
\hline Variable & Modalités & Fréquence & Pourcentage \\
\hline \multirow{3}{*}{$\begin{array}{l}\text { Sources de revenu } \\
\text { du ménage }\end{array}$} & La première source de revenu du ménage & 163 & 90,6 \\
& est l'agriculture & & \\
& C'est une source autre que l'agriculture & 16 & 8,9 \\
& Total & $\mathbf{1 7 9}$ & $\mathbf{9 9 , 4}$ \\
\hline \multirow{3}{*}{$\begin{array}{l}\text { Contribution du } \\
\text { palmier à huile au }\end{array}$} & {$[2-5] \%$} & 64 & 35,8 \\
revenu du ménage & {$[51-75] \%$} & 22 & 12,3 \\
& {$[76-100] \%$} & 26 & 14,5 \\
& Total $\%$ & 67 & 37,4 \\
& Magasins de vente des intrants agricoles & $\mathbf{1 7 9}$ & $\mathbf{1 0 0}$ \\
& Financement des activités agricoles & 24 & 21,1 \\
& Huileries & 10 & 13,3 \\
Contraintes dans la & Outil agricole & 12 & 5,6 \\
zone d'étude & Cestion de l'exploitation & 10 & 6,7 \\
& Approvisionnement en matériel végétal & 14 & 5,6 \\
& amélioré du palmier à huile & 16 & 7,8 \\
& Services d'encadrement technique & & 8,9 \\
& Réponses manquantes & 21 & 11,7 \\
& Total & 35 & 19,4 \\
& & $\mathbf{1 8 0}$ & $\mathbf{1 0 0 , 0}$ \\
\hline
\end{tabular}




\begin{tabular}{llcc}
\hline & Meilleur rendement en huile végétale & 16 & 8,9 \\
& Besoins de consommation et de & 39 & 21,7 \\
& commercialisation & & \\
Motivation à la & Disponibilité des terres & 2 & 1,1 \\
culture du palmier à & Présence de la SOCAPALM & 1 & 0,6 \\
huile & Imitation des autres producteurs & 1 & 0,6 \\
& Proximité d'une huilerie & 12 & 6,7 \\
& Culture rentable & 2 & 1,1 \\
& Autres & 4 & 2,2 \\
& Réponses manquantes & 103 & 57,2 \\
& Total & $\mathbf{1 8 0}$ & $\mathbf{1 0 0 , 0}$ \\
\hline
\end{tabular}

Source : Données d'enquête des ménages, 2009.

\section{Conclusion}

Loin d'être une comparaison entre les différentes causes de déforestation dans le bassin du Congo, cette étude a voulu juste montrer les effets de certaines pratiques agricoles extensives et particulièrement de défriche-brulis très consommatrice d'espace, sur la destruction du couvert forestier, malgré les effets bénéfiques chez les populations pauvres. Ainsi, la poursuite des objectifs d'accroissement de la production d'huile de palme au Cameroun, devrait tenir en compte les conséquences environnementales pour éviter certains problèmes qui sont déjà observés dans d'autres pays tropicaux grands producteurs d'huile de palme comme l'Indonésie, la Malaise, la Nouvelle Papouasie.

Nous recommandons pour la relance de l'élaéiculture familiale au Cameroun, que les jeunes qui sont plus nombreux, soient encouragés à entrer dans la filière de production grâce à une politique agricole adéquate. Ceci peut se faire en créant des huileries dans les différents bassins de production, en assurant un meilleur approvisionnement en intrants agricole, en créant un système de crédit agricole efficace et en assurant un encadrement technique adéquat aux producteurs. Cependant, l'État camerounais devrait être nanti d'outils fiables de prise de décision stratégiques à l'instar des modèles permettant d'analyser les dynamiques des systèmes agraires. De tels modèles permettraient de réguler l'expansion de la palmeraie familiale en essayant de trouver un équilibre, comme l'optimum de Pareto (Jacquemin and Tulkens, 1996), entre l'augmentation de la production nationale d'huile de palme et la protection des ressources naturelles; ce qui permettrait au pays de participer à la lutte contre le changement climatique dans le bassin du Congo.

\section{REMERCIEMENTS}

Nous tenons à remercier vivement toute l'équipe dirigeante du projet de Renforcement des Capacités dans la Recherche Agricole au Cameroun (REPARAC), pour son soutien technique et financier ayant favorisé la collecte et le traitement des données qui ont permis d'écrire cet article. Nous remercions également tous les relecteurs pour leurs brillants commentaires.

\section{REFERENCES}

Amougou JA. 2010. Le REDD au Cameroun : contexte actions et perspectives. Ministère de l'Environnement et de la Protection de la Nature. CCNUCC, Yaoundé - Cameroun.

Besley T, Case A. 1993. Modeling technology adoption in developing countries. The American Economic Review, 83(2): 396402.

BUCREP. 2010. Troisième Recensement de la Population et de l'Habitat. BUCREP, Yaoundé, 67 p. 
Caliendo M, Hujer R. 2005. The microeconometric estimation of treatment effects - an overview. Discussion paper No. 1653. Forschungs institut, zur Zukunft der Arbeit, Institute for the Study of Labor.

Delacote PH. 2008. La pauvreté, terreau de la déforestation. Alternatives Economiques, $\mathrm{n}^{\circ} 265$.

Diagne A, Demont M. 2007. Taking a new look at empirical models of adoption: average treatment effect estimation of adoption rates and their determinants. Agricultural Economics, 37: 201-210.

Dimara E, Skuras D. 2003. Adoption of agricultural innovations as a two-stage partial observability process. Agric. Econ., 28: 187-196.

Doumenge C. 1998. La gestion des écosystèmes forestiers du Cameroun, du Gabon et de Guinee équatoriale a l'aube de l'an 2000. UICN, Yaoundé, Cameroun.

Estrada PM, Corbera E, Brown K. 2007. Reducing greenhouse gas emissions from deforestation in developing countries: revisiting the assumptions. Tyndall Centre for Climate Change Research, Working Paper $\mathrm{N}^{\circ} 115$. Norwich, UK.

FAO 2007. Situation des forêts du monde en 2007, Rome.

Fitzherbert EB, Struebig MJ, Morel A, Danielsen F, Brühl CA, Donald PF, Phalan B. 2008. How will palm oil expansion affect biodiversity? Trends in Ecology and Evolution, 23(10): 538-545.
Jacquemin A, Tulkens H. 1996. Fondements d'Economie Politique ( $2^{\text {nd }}$ edn). De Boeck: Bruxelles; 213-215.

Kanninen M, Murdiyarso D, Seymour F, Angelsen A, Wunder S, German L. 2007. Do trees grow on money? The implications of deforestation research for policies to promote REDD. Forest Perspectives No. 4. Bogor, Indonésie.

Lebailly P, Tentchou J. 2009. Étude sur la filière porteuse d'emplois «palmier à huile ». Rapport final : Projet d'appui à la promotion de l'emploi et réduction de la pauvreté. Ministère de l'Emploi et de la Formation Professionnelle et OIT Bureau Sous-Régional de l'Afrique Centrale. Yaoundé, Cameroun.

Otsuka K, Place F. 2001. Land Tenure and Natural Resource Management: A Comparative Study of Agrarian Communities in Asia and Africa. John Hopkins University Press: Baltimore.

Rossi G, André V. 2006. La biodiversité : questions de perspectives. Annales de Géographie, 115(651).

Sascha OB. Andres A. 2002. Estimation of average treatment effects based on propensity scores. The Stata Journal, 4: 358-377.

UNEXPALM. 2010. Rapport annuel d'activité. MINADER. Yaoundé, Cameroun.

World Rainforest Movement. 2007. Transgenic Trees. Montevideo, WRM Briefing. 